APS

physics

This is the accepted manuscript made available via CHORUS. The article has been published as:

\title{
Rotation Reversal Bifurcation and Energy Confinement Saturation in Tokamak Ohmic L-Mode Plasmas
}

J. E. Rice, I. Cziegler, P. H. Diamond, B. P. Duval, Y. A. Podpaly, M. L. Reinke, P. C. Ennever, M. J. Greenwald, J. W. Hughes, Y. Ma, E. S. Marmar, M. Porkolab, N. Tsujii, and S. M. Wolfe Phys. Rev. Lett. 107, 265001 - Published 20 December 2011 DOI: 10.1103/PhysRevLett.107.265001 


\title{
Rotation Reversal Bifurcation and Energy Confinement Saturation in Tokamak Ohmic L-mode Plasmas
}

\author{
J.E. Rice, I. Cziegler, P.H. Diamond ${ }^{\ddagger}$, B.P. Duval ${ }^{\dagger}$, Y.A. Podpaly, M.L. Reinke, \\ P.C. Ennever, M.J. Greenwald, J.W. Hughes, Y. Ma, E.S. Marmar, M. Porkolab, \\ N. Tsujii and S.M. Wolfe \\ PSFC, MIT, Cambridge, Massachusetts 02139, USA \\ ${ }^{\ddagger}$ UCSD, San Diego, California 92903, USA and NFRI, Daejeon 305-333, Korea \\ ${ }^{\dagger}$ CRPP, EPFL, Lausanne 1015, Switzerland
}

\begin{abstract}
Direction reversals of intrinsic toroidal rotation have been observed in diverted Alcator C-Mod Ohmic L-mode plasmas following electron density ramps. For low density discharges, the core rotation is directed co-current, and reverses to counter-current following an increase in the density above a certain threshold. Such reversals occur together with a decrease in density fluctuations with $2 \mathrm{~cm}^{-1} \leq \mathrm{k}_{\theta} \leq 11 \mathrm{~cm}^{-1}$ and frequencies above $70 \mathrm{kHz}$. There is a strong correlation between the reversal density and the density at which the Ohmic L-mode energy confinement changes from the linear to the saturated regime.
\end{abstract}


Plasma rotation due to self-acceleration by turbulence is one example of a class of macroscopic self-organization problems that includes the origin of solar differential rotation, the geodynamo and the general circulation of the ocean. Spontaneous inversions in self-accelerated toroidal flows in tokamaks will be covered here. Self-generated flow in H-mode and other enhanced confinement regimes is generally directed co-current and has been found to have a relatively simple global scaling [1], with the Mach number proportional to the plasma pressure. In contrast, the intrinsic rotation in Ohmic L-mode plasmas has a complicated dependence on global plasma parameters [2, 3]. In fact, rotation inversions or reversals have been observed $[4,5,3]$, where the core rotation abruptly switches direction, with negligible effect on other macroscopic plasma parameters. Rotation inversions constitute a novel form of momentum transport bifurcation. Rotation reversals have been induced with changes in density, plasma current and magnetic field. A seemingly unrelated phenomenon, which is a long standing mystery, concerns the progression from the linear Ohmic confinement (LOC) regime to the saturated Ohmic confinement (SOC) regime. This transition occurs at a particular density $[6,7,8]$ depending on machine and plasma parameters. In this paper, the connection among rotation reversals, the transformation from LOC to SOC, and changes in turbulence characteristics is examined. All three of these phenomena are observed to occur at the same critical density.

A detailed study of intrinsic rotation reversals in Ohmic L-mode plasmas has been performed [9] on the Alcator C-Mod tokamak [10] (major radius $\mathrm{R}=0.67 \mathrm{~m}$, typical minor radius of $0.21 \mathrm{~m}$ ). One method of inducing reversals is by ramping the electron density $[4,5,3]$. The reversal process is quite sensitive to the value of the electron density, which is demonstrated in Fig.1. As can be seen by the solid line in the top frame, this plasma experienced two reversals, first from the counter- to co-current direction begining at $0.666 \mathrm{~s}$, following a slight decrease in the electron density, and from co- to counter-current initiating at $1.146 \mathrm{~s}$, with a slight density increase. Positive $\mathrm{V}_{\text {Tor }}$ denotes co-current rotation. These rotation changes with density are the same as was seen in TCV diverted discharges, but opposite to limited plasmas [4, 5]. During the reversals, the density profile shape did not change. The overall hysteresis cycle transpired with a $10 \%$ change in the electron density. The existence of a hysteresis cycle is prima facie evidence that the inversion is in fact a bifurcation and a first order transition. The magnitude of the core rotation excursion, determined from the Doppler shifts of argon $\mathrm{x}$-ray lines (which is representative of the bulk ion flow), was $\sim 30 \mathrm{~km} / \mathrm{s}$. Reversals usually evolve on a time scale comparable to the momentum confinement time [11] and occur inside of a particular radius, near the $\mathrm{q}=3 / 2$ surface [9]. For the discharge of Fig.1, the reversal developed inside of $\mathrm{R}=0.84 \mathrm{~m}(\mathrm{r} / \mathrm{a}=0.75)$, with no change in the rotation profile outside of that location, as can be seen by the dashed line in the top frame. There is a transient change in the rotation in the opposite direction at the plasma edge which might play a role in momentum conservation [9]. The total torque density required to reverse the rotation in the core was $\sim 0.4 \mathrm{~N} / \mathrm{m}^{2}$. This is similar in magnitude to the measured Reynolds stress torque in DIII-D plasmas [12].

The density at which the reversal occurs depends on the plasma current. A large body of C-Mod rotation reversal data is summarized in Fig.2 where the line average electron density at the time when the central rotation begins to reverse is plotted as a function of plasma current for $5.4 \mathrm{~T}$ discharges. This covers a range of $\mathrm{q}_{95}$ from 3.0 


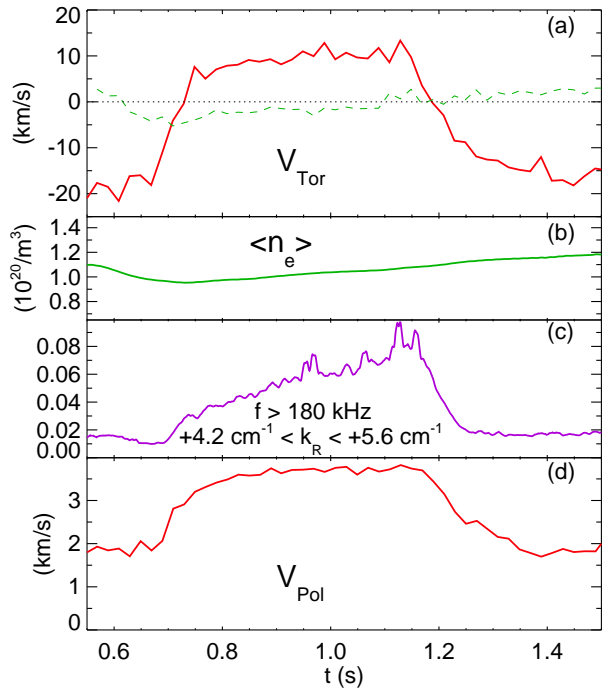

Figure 1: Time histories of (a) the toroidal rotation velocity in the center (solid) and outside of $\mathrm{r} / \mathrm{a}=0.75$ (dashed), (b) average electron density, (c) density fluctuation intensity with $\mathrm{k}_{R}$ between 4.2 and $5.6 \mathrm{~cm}^{-1}$ and frequency above $180 \mathrm{kHz}$ and (d) the poloidal propagation velocity of the turbulence for a $1.1 \mathrm{MA}, 5.4 \mathrm{~T}\left(\mathrm{q}_{95}=3.2\right)$ discharge which underwent two rotation reversals. 


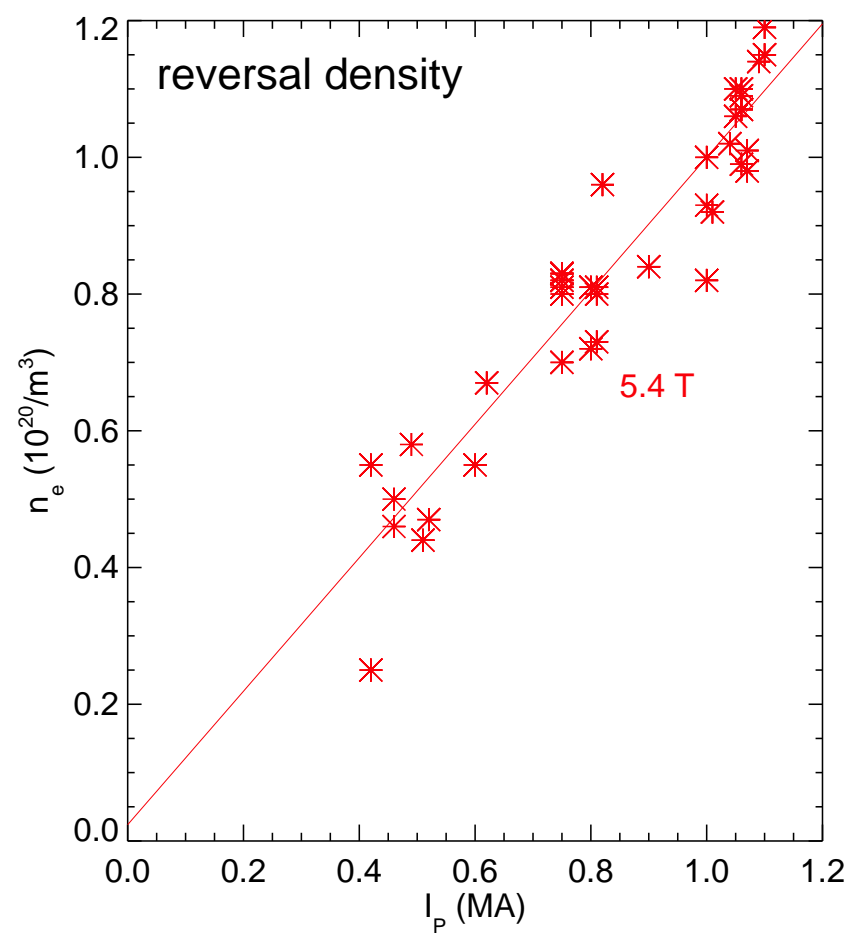

Figure 2: The density at the rotation reversal initiation time as a function of plasma current for $5.4 \mathrm{~T}$ discharges. The line represents the best linear fit. 
to 7.0. There is a linear increase of the reversal density with plasma current over a span of a factor of three. Some of the scatter is because plasmas with both upward and downward density ramps have been included. As was seen in Fig.1, there is a hysteresis of about $10 \%$ in density.

There is very little change $[4,5,9]$ in the density, temperature or q profiles (and other macroscopic parameters) during the reversal process, while there is a drastic transformation in the toroidal velocity profiles inside of the $q=3 / 2$ surface. There are also significant changes in the character of density fluctuations. Shown in Fig. 3 are dispersion plots of density fluctuations from the phase contrast imaging (PCI) diagnostic [13] before $(0.608 \mathrm{~s})$ and after $(0.859 \mathrm{~s})$ the first core reversal of the discharge shown in Fig.1. The PCI signal is provided by a continuous $\mathrm{CO}_{2}$ laser beam which passes
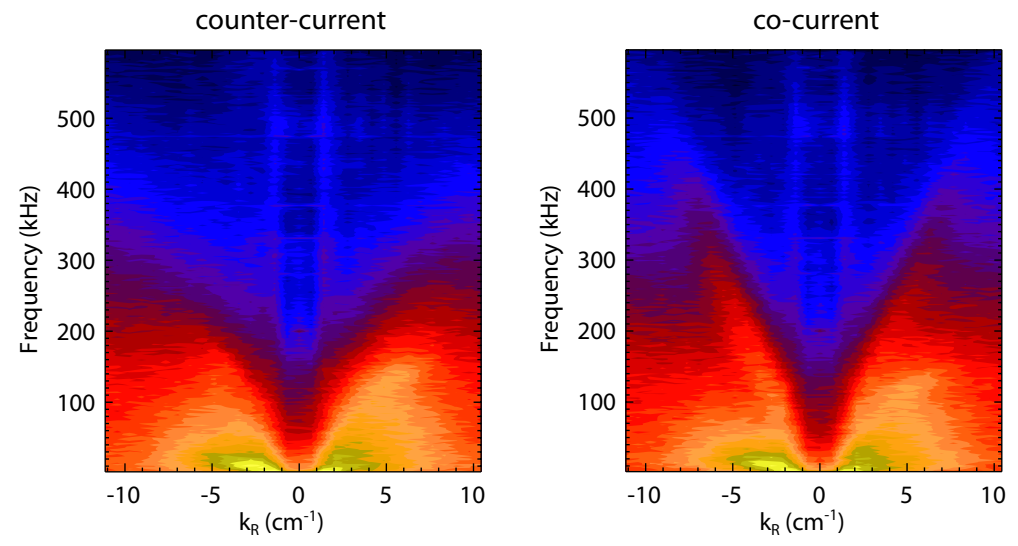

Figure 3: Dispersion plots $\mathrm{S}(\mathrm{k}, \mathrm{f})$ of turbulence before $(0.608 \mathrm{~s}$, counter-current rotation, left panel $)$ and after $(0.859 \mathrm{~s}$, co-current rotation, right panel) the first rotation reversal in the discharge shown in Fig.1.

through the plasma vertically. The PCI technique is sensitive to fluctuation spectra with a finite wavenumber perpendicular to the beam, i.e. to the component of $\mathrm{k}_{R}$ along the major radius. There are distinct lobes with $\left|\mathrm{k}_{R}\right| \geq 2 \mathrm{~cm}^{-1}$ for frequencies above 100 $\mathrm{kHz}$ which are apparent when the rotation is directed co-current $\left(\mathrm{n}_{e}<1 \times 10^{20} / \mathrm{m}^{3}\right)$ but 
which are not present when the rotation is counter-current $\left(\mathrm{n}_{e}>1 \times 10^{20} / \mathrm{m}^{3}\right)$. The correlation between the appearance of these features and the direction of the core toroidal rotation velocity is clearly demonstrated in the third frame of Fig.1, which shows the time history of fluctuation intensity with $\mathrm{k}_{R}$ between +4.2 and $+5.6 \mathrm{~cm}^{-1}$ and with frequencies above $180 \mathrm{kHz}$. The total power in the density fluctuation signal (normalized to the average electron density) is clearly observed to increase when the fast lobes are present. Shown in Fig. 4 is the conditional spectrum $S(k, f) / S(f)$ of the difference between spectrograms at $0.859 \mathrm{~s}$ (co-current) and $0.608 \mathrm{~s}$ (counter-current), which demonstrates the distinct structure of the lobes, with $\left|\mathrm{k}_{R}\right|$ extending from 2 to above $11 \mathrm{~cm}^{-1}$ for frequencies greater than $70 \mathrm{kHz}$. The slope of these features indicates

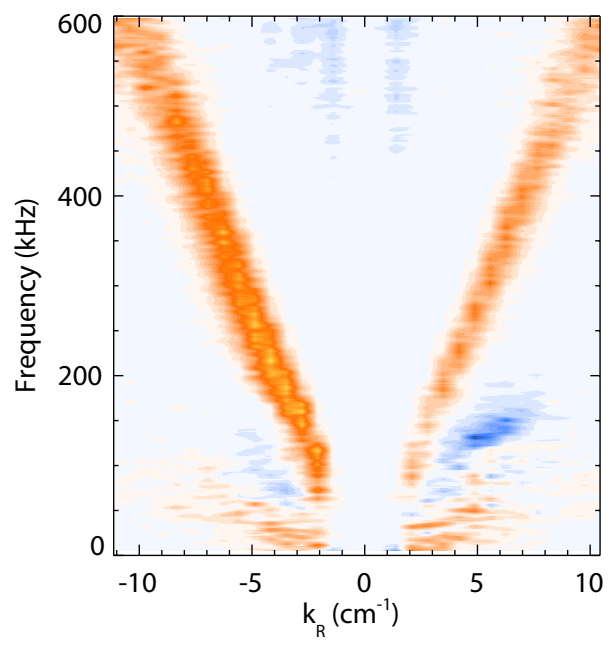

Figure 4: Conditional spectrum, $\mathrm{S}(\mathrm{k}, \mathrm{f}) / \mathrm{S}(\mathrm{f})$, of the difference between dispersion plots from $0.859 \mathrm{~s}(\mathrm{co}-)$ and $0.608 \mathrm{~s}$ (counter-).

a poloidal phase velocity of $\mathrm{V}_{\theta}=\omega / \mathrm{k}_{\theta}=2 \pi \mathrm{f} / \mathrm{k}_{R} \sin \zeta \sim 3.8 \mathrm{~km} / \mathrm{s}$, where $\zeta$ is the angle the PCI viewing chord makes with the magnetic flux surface at the location of the measurement $\left(\mathrm{k}_{\theta}=\mathrm{k}_{R} \sin \zeta\right.$ owing to the reciprocal vector nature of the wavenumber). Both positive and negative $\mathrm{k}_{R}$ spectral lobes speed up simultaneously in both propagation directions. This strongly suggests that (due to the line integration) these lobes are collected from two spatial locations along the PCI chord, from the top and bottom 
of a flux surface, where they suffer the same Doppler shift caused by the local radial electric field. Since the slopes (phase velocities) of the two lobes are the same (within $5 \%$ ), this suggests that the poloidal flux surface where the signal originates is up-down symmetric to within a similar degree. For this discharge, with an upper triangularity of 0.30 and a lower triangularity of 0.44 at the separatrix, this implies that the lobes reside inside of $\mathrm{R}=0.84 \mathrm{~m}(\mathrm{r} / \mathrm{a}=0.75, \sin \zeta \geq 0.96)$, which is also inside of the reversal radius. These features were not observed with gas puff imaging or reflectometry at the plasma edge, providing further evidence that the relevant changes in turbulence are in the plasma core. The time evolution of the poloidal propagation velocity of the lobes is shown in the bottom frame of Fig. 1 and is well correlated with the co-current core toroidal rotation speed. For these features, $\mathrm{k}_{\theta} \rho_{s}$ is between $0.15-0.7$, which is in the range of trapped electron mode (TEM) turbulence. Fluctuations originating from the top or the bottom of the plasma may be isolated with the PCI diagnostic [13] through the use of masking. By utilization of this technique, it was determined that these features propagate in the ion diamagnetic drift direction in the laboratory reference frame. However, due to substantial Doppler shift from plasma rotation in the ion direction, the propagation direction of these features in the plasma frame is unknown.

In order to address the question of what can cause the toroidal rotation to switch direction while other macroscopic parameters remain unchanged, it is informative to examine the momentum flux. The momentum flux is proportional to the Reynolds stress, which can be written as the sum of three terms [14]

$$
-\chi_{\phi} \partial \mathrm{v}_{\phi} / \partial \mathrm{r}+\mathrm{V}_{P} \mathrm{v}_{\phi}+\Pi^{\text {res }}
$$

respectively proportional to the momentum diffusivity $\chi_{\phi}$, the momentum pinch $\mathrm{V}_{P}$ and the residual stress $\Pi^{\text {res }}$. To see that the Reynolds stress determines the momentum flux, note that the convective flux $\left(\sim\left\langle\tilde{V}_{r} \tilde{n}\right\rangle\left\langle V_{\phi}\right\rangle\right)$ is necessarily vanishing in the absence of core particle sources. Neoclassical contributions to the momentum flux are well known to be negligible and higher order nonlinearities $\left(\sim\left\langle\tilde{n} \tilde{V}_{r} \tilde{V}_{\phi}\right\rangle\right)$ are subdominant. The Maxwell stress and finite $\beta$ effects have been ignored since $\beta_{N} \leq 0.29$ for these plasmas. A reversal in intrinsic rotation requires either a change in sign of $\mathrm{V}_{P} \mathrm{v}_{\phi}$ at the last closed flux surface or a change in sign of the residual stress, and the resulting intrinsic torque, $-\partial_{r} \Pi^{r e s}$. ( $\chi_{\phi}$ is positive definite and cannot change sign.) For both ion temperature gradient (ITG) and TEM instabilities, the turbulent equipartition pinch [15] is directed inward and cannot change sign. An additional thermoelectric pinch is comprised of oppositely directed $\nabla \mathrm{n}$ and $\nabla \mathrm{T}_{i}$ pieces and thus is usually negligible [16]. In addition, the edge $\nabla \mathrm{n}$ and $\nabla \mathrm{T}_{i}$ do not change during the reversal process. Similarly, the sign of the Coriolis pinch [17] can change only if the density profile gradient changes sign. The density gradient definitely does not change sign following the rotation reversal [9], so the flow pinch most likely does not play a role in the reversals. The residual stress can change sign depending on the nature of the underlying turbulence, such as by a switch in the mode propagation direction. This is becasue the sign of the residual stress, and thus that of the intrinsic torque, are predicted to flip when the propagation direction of the turbulence in the plasma frame changes [18]. One example would be a change of wave propagation from the electron diamagnetic drift (EDD) direction (electron drift waves or TEMs) to the ion diamagnetic drift (IDD) direction (ion drift waves or ITG modes) as the density exceeds a critical threshold [18]. A switch 
in the turbulence propagation from the EDD to the IDD direction has in fact been observed in Alcator C discharges [19] at a particular density, and such a change from TEM (or ETG) to ITG turbulence domination has been invoked to explain the transition in global energy confinement from the linear (LOC, neo-Alcator) to the saturated Ohmic confinement regime [19]. Similarly, the appearance of ion diamagnetic drift direction propagating turbulence has been observed in TEXT [20], DIII-D [21] and ASDEX Upgrade [22] plasmas following the transformation from LOC to SOC. Interestingly, the reversal density of $\sim 0.8 \times 10^{20} / \mathrm{m}^{3}$ for $5.4 \mathrm{~T}, 0.8$ MA C-Mod discharges (Fig.2) is very close to the density separating the linear energy confinement regime from the saturated confinement regime for Ohmic discharges (Fig.1 of [8]). This connection is emphasized in Fig.5 which shows (a) the global energy confinement time (from magnetics) and (b) the core toroidal rotation velocity as a function of electron density for a series of individual $0.62 \mathrm{MA}, 5.2 \mathrm{~T}$ discharges. The density of the rotation reversal is very

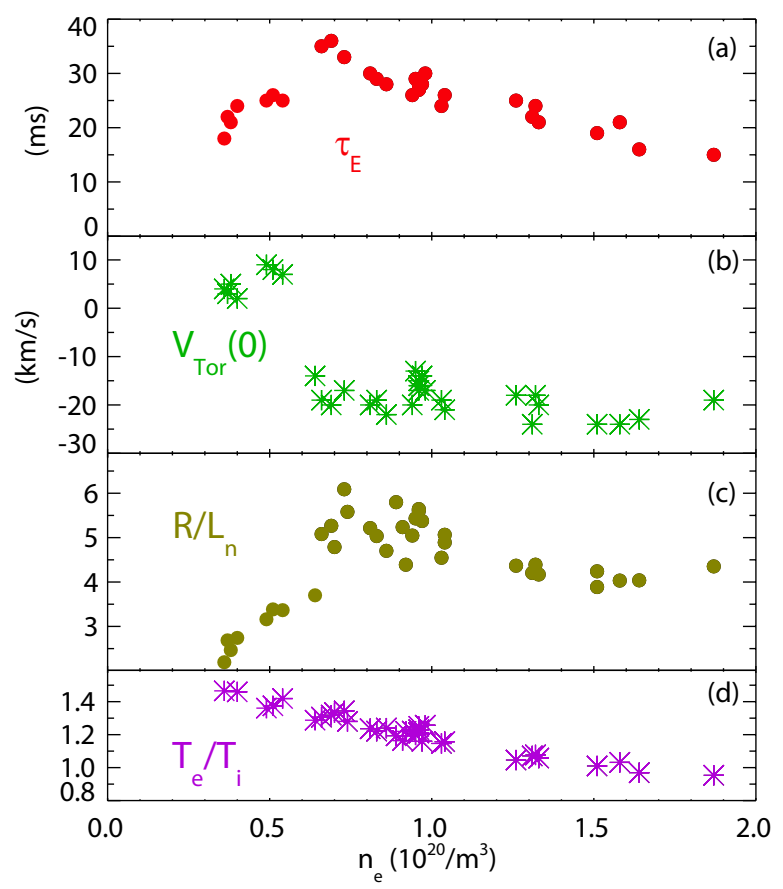

Figure 5: The global energy confinement time (a), central toroidal rotation velocity (b), inverse density gradient scale length at $\mathrm{R}=0.79 \mathrm{~m} \mathrm{(c)}$ and the central $\mathrm{T}_{e} / \mathrm{T}_{i}$ ratio (d) as a function of average electron density for a series of $0.62 \mathrm{MA}, 5.2 \mathrm{~T}\left(\mathrm{q}_{95}=5.0\right)$ discharges.

close to the density which separates the linear increase in global energy confinement 
from the saturated confinement regime, around $0.6 \times 10^{20} / \mathrm{m}^{3}$ for these operating conditions (see Fig.2). Changes in particle transport also occur at this density. R/L $\mathrm{L}_{n}$, the inverse density gradient scale length (c) from Thomson scattering profiles (evaluated at $\mathrm{R}=0.79 \mathrm{~m}$, inside of the rotation reversal radius for these discharge conditions [9]) also makes an abrupt change at the same density. In the LOC regime, below $0.6 \times 10^{20} / \mathrm{m}^{3}$, the density profile peaks up with increasing electron density; in the SOC regime, the density profile shape remains constant with increasing density. Over this density range, the ratio (d) between the electron and ion temperatures smoothly varies, indicative of an increase in ion heating due to stronger collisional transfer from electrons to ions. At the reversal density the ratio of $\mathrm{T}_{e} / \mathrm{T}_{i}$ at the plasma center changed from $\geq 1.35$ (at low density) to $\leq 1.35$ (at high density), but in a continuous fashion.

A comparison of the rotation reversal density and the transition density from the linear to saturated Ohmic confinement regimes for several discharge conditions is shown in Fig.6. There is an excellent correlation between these two quantities over a factor

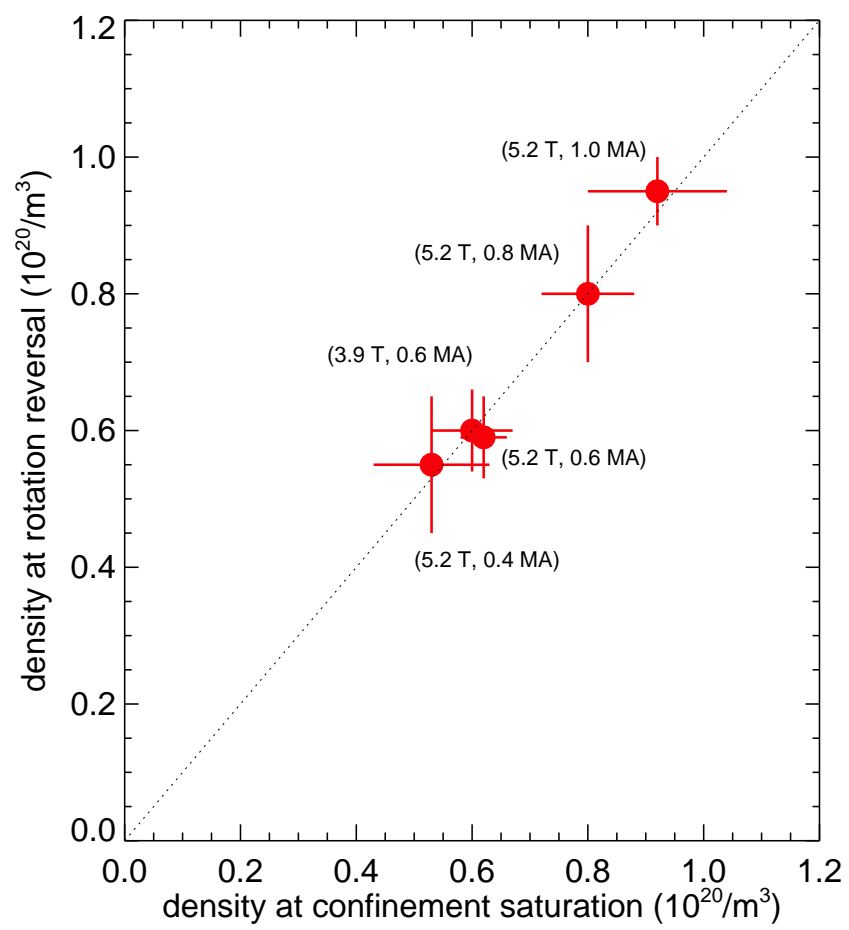

Figure 6: The rotation reversal density as a function of the transition density between linear and saturated energy confinement. Magnetic fields and plasma currents for each point are listed. The dotted line has a slope of unity.

of two in density. The connection among rotation reversals, Ohmic confinement satu- 
ration, density profile peaking and turbulence changes can be unified by the following ansatz. At low density, where the core toroidal rotation is in the co-current direction, and where Ohmic energy confinement, coupling between electrons and ions, and density profile peaking all increase with increasing density, a turbulence feature with $\mathrm{k}_{\theta}$ between 2 and $11 \mathrm{~cm}^{-1}$, consistent in nature with TEM instabilities, is present. At a critical density, this high $\mathrm{k}$ turbulent feature disappears, the core rotation reverses due to a change in sign of the intrinsic torque $\left(-\partial_{r} \Pi^{r e s}\right)$, and global energy confinement and density profile peaking saturate, possibly due to a change to predominance of ITG modes in the turbulence spectrum. This scenario is consistent with previous observations on Alcator C, TEXT, DIII-D and ASDEX Upgrade (but with the addition of rotation measurements on C-Mod).

In summary, direction reversals of intrinsic toroidal rotation have been observed in diverted Alcator C-Mod Ohmic L-mode plasmas following modest electron density ramps. The reversal occurs in the plasma interior, inside of the $q=3 / 2$ surface. For low density plasmas, the rotation is in the co-current direction, and reverses to the counter-current direction following an increase in the electron density above a certain threshold. Reversals from the co- to counter-current direction are correlated with a sharp decrease in density fluctuations with $2 \mathrm{~cm}^{-1} \leq \mathrm{k}_{\theta} \leq 11 \mathrm{~cm}^{-1}$ and frequencies above $70 \mathrm{kHz}$. For low density operation with co-current rotation, this corresponds to the conditions where TEMs are expected to be excited. The density at which the rotation reverses increases linearly with plasma current. There is a strong correlation between the reversal density and the density at which the global Ohmic L-mode energy confinement changes from linear to saturated.

The authors thank J.Terry, J.Dorris, D.Ernst and W.Xiao for enlightening discussions, J.Irby for electron density measurements and the Alcator C-Mod operations group for expert running of the tokamak. Work supported at MIT by DoE Contract No. DE-FC02-99ER54512.

\section{References}

[1] J.E.Rice et al., Nucl. Fusion 47 (2007) 1618.

[2] J.E.Rice et al., Nucl. Fusion 45 (2005) 251.

[3] J.E.Rice et al., Plasma Phys. Contr. Fusion 50 (2008) 124042.

[4] A.Bortolon et al., Phys. Rev. Lett. 97 (2006) 235003.

[5] B.P.Duval et al., Plasma Phys. Contr. Fusion 49 (2007) B195.

[6] R.R.Parker et al., Nucl. Fusion 25 (1985) 1127.

[7] F.X.Söldner et al., Phys. Rev. Lett. 61 (1988) 1105.

[8] L.Lin et al., Plasma Phys. Contr. Fusion 51 (2009) 065006.

[9] J.E.Rice et al., Nucl. Fusion 51 (2011) 083005. 
[10] E.S.Marmar et al., Fusion Sci. Technol. 51 (2007) 261.

[11] W.D.Lee et al., Phys. Rev. Lett. 91 (2003) 205003.

[12] S.H.Müller et al., Phys. Rev. Lett. 106 (2011) 115001.

[13] M.Porkolab et al., I.E.E.E. Trans. Plasma Sci. 34 (2006) 229.

[14] P.H.Diamond et al., Nucl. Fusion 49 (2009) 045002.

[15] T.S.Hahm et al., Phys. Plasmas 14 (2007) 072302.

[16] E.S.Yoon and T.S.Hahm, Nucl. Fusion 50 (2010) 064006.

[17] A.Peeters et al., Phys. Rev. Lett. 98 (2007) 265003.

[18] P.H.Diamond et al., Phys. Plasmas 15 (2008) 012303.

[19] R.L.Watterson et al., Phys. Fluids 28 (1985) 2857.

[20] D.L.Brower et al., Phys. Rev. Lett. 59 (1987) 48.

[21] C.L.Rettig et al., Phys. Plasmas 8 (2001) 2232.

[22] G.D.Conway et al., Nucl. Fusion 46 (2006) S799. 


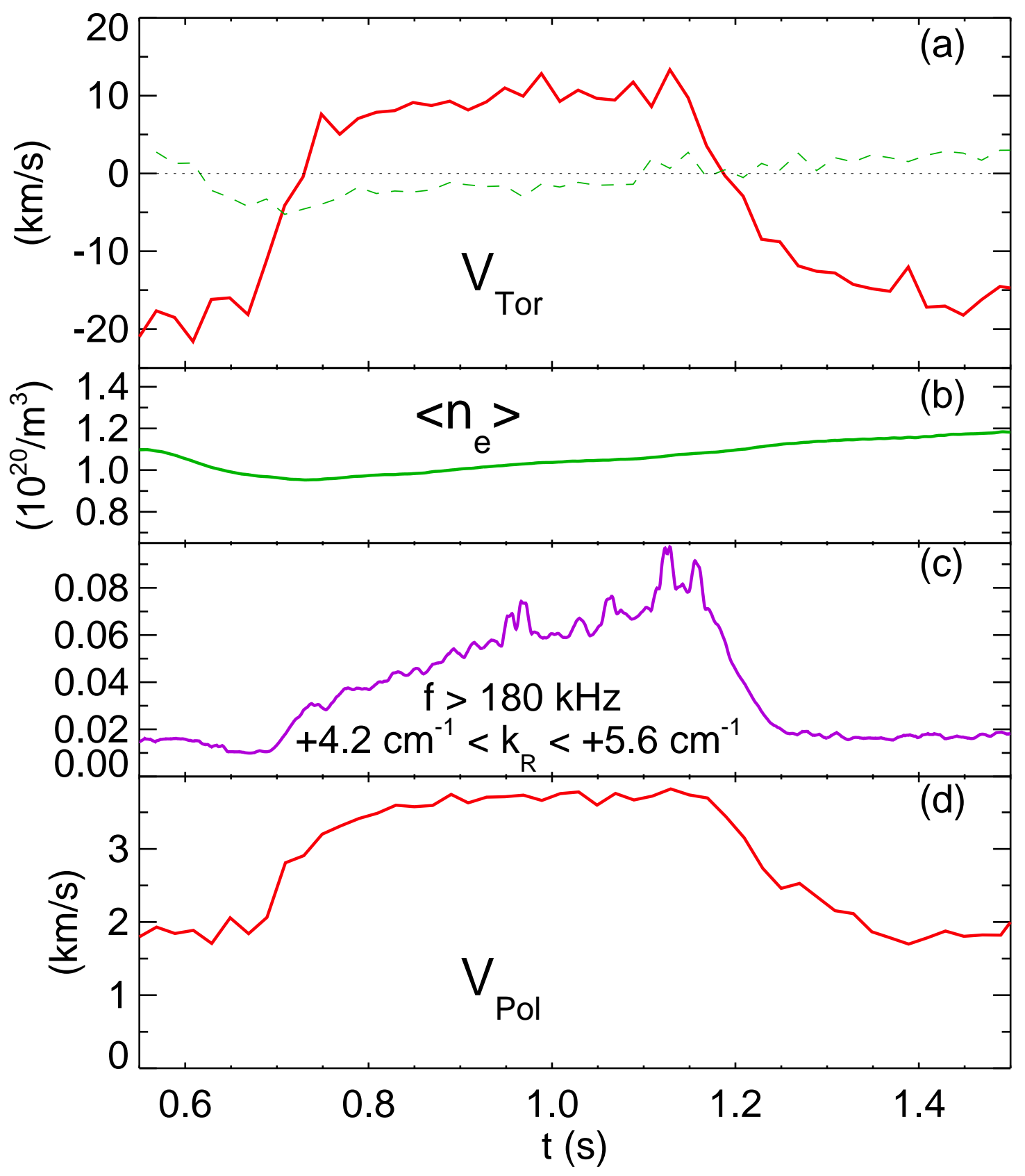

Figure $01 \quad$ LE13125 22Nov2011 


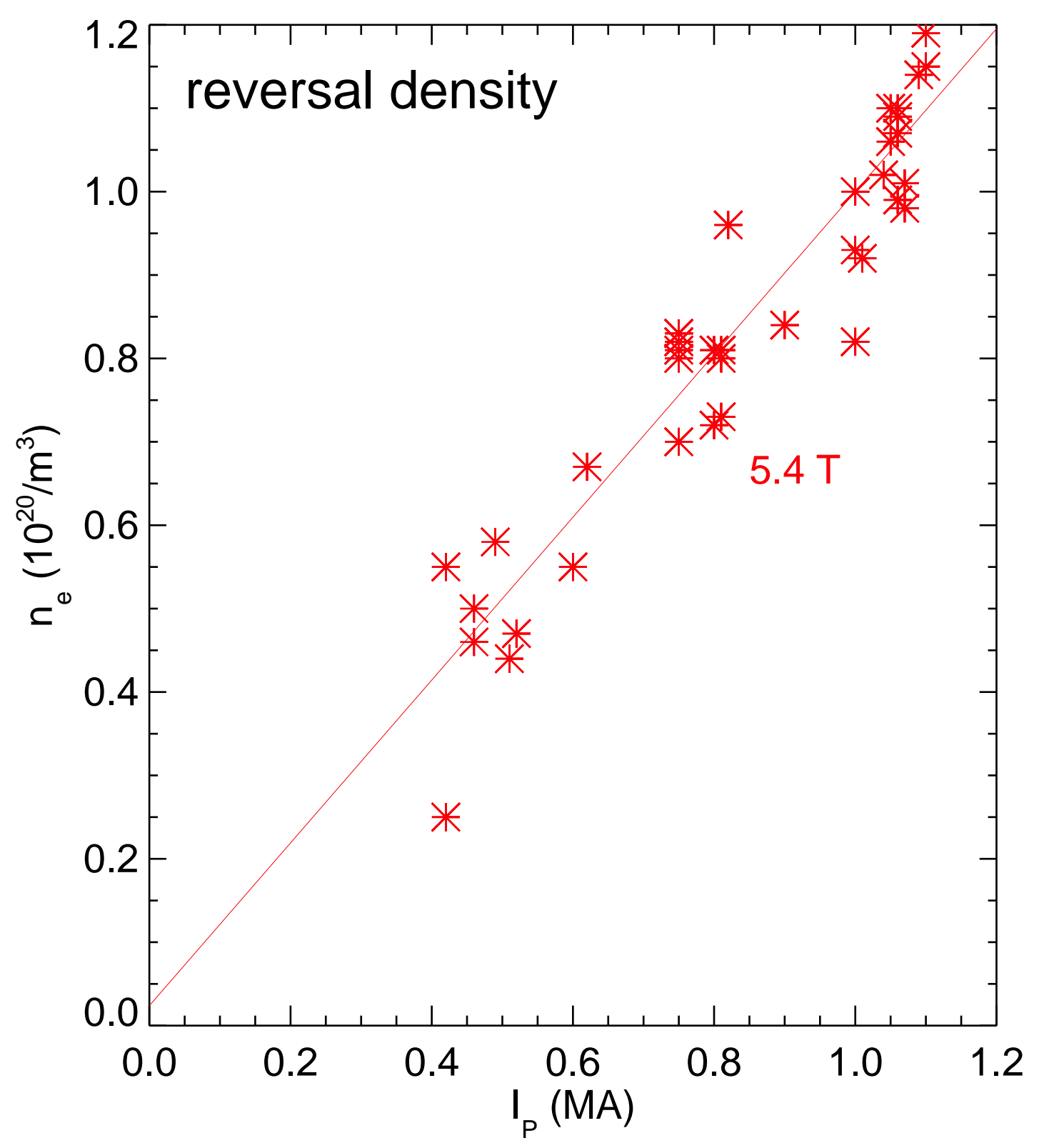

Figure $02 \quad$ LE13125 22Nov2011 

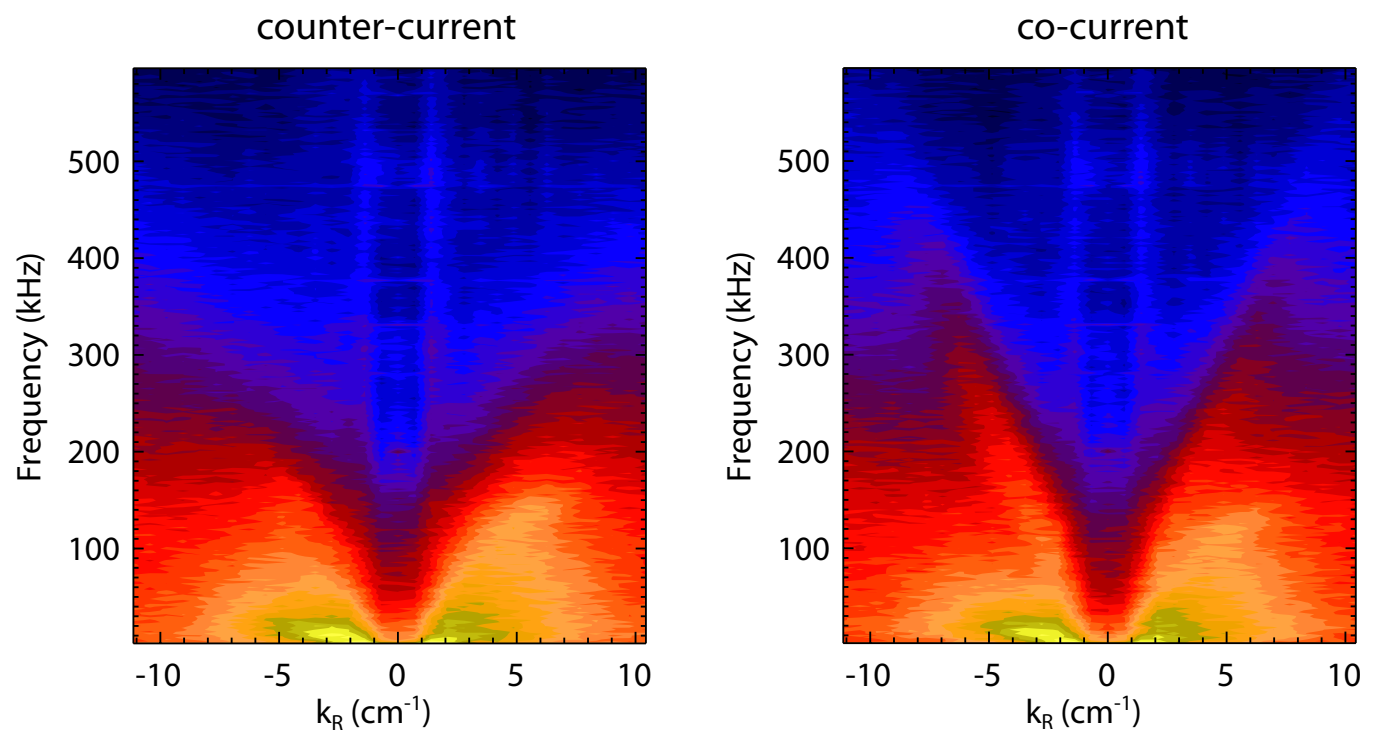

Figure $03 \quad$ LE13125 22Nov2011 


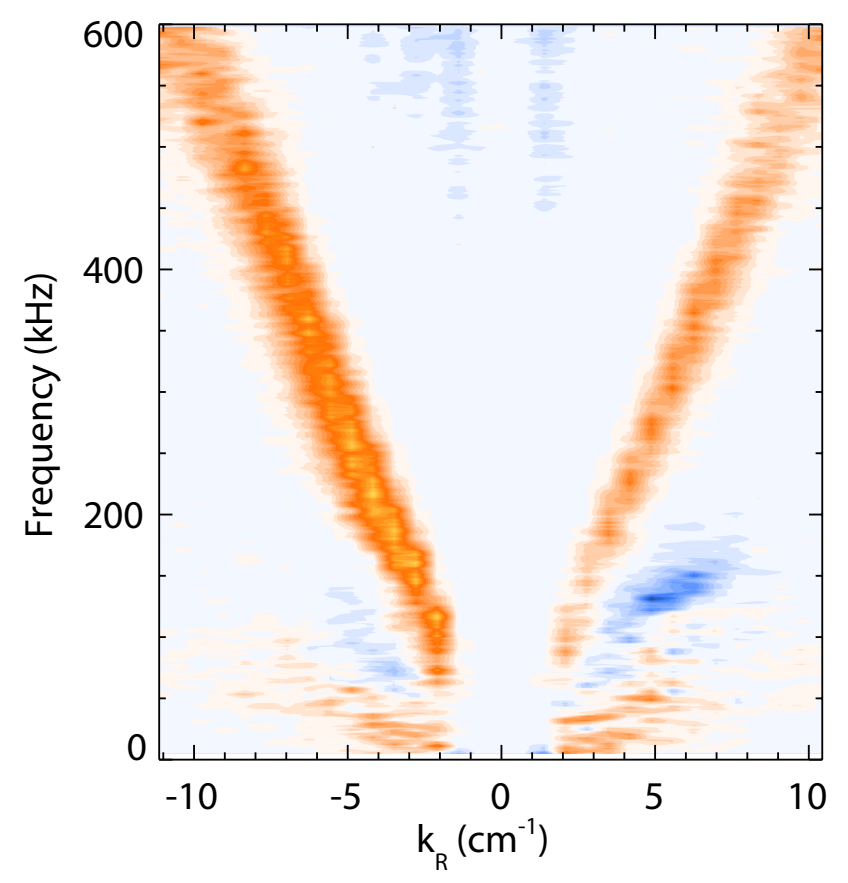

Figure $04 \quad$ LE13125 22Nov2011 


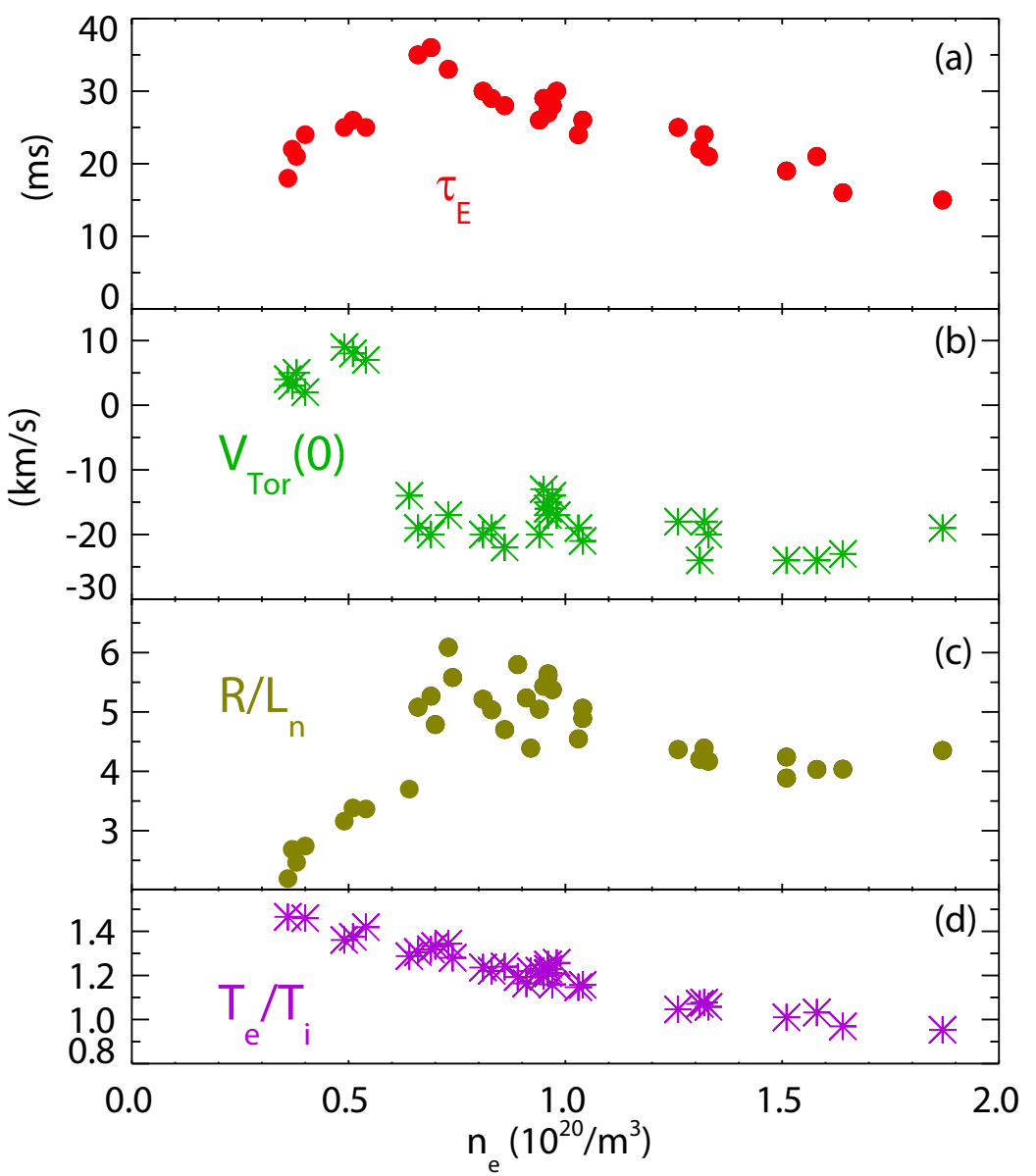

Figure $05 \quad$ LE13125 22Nov2011 


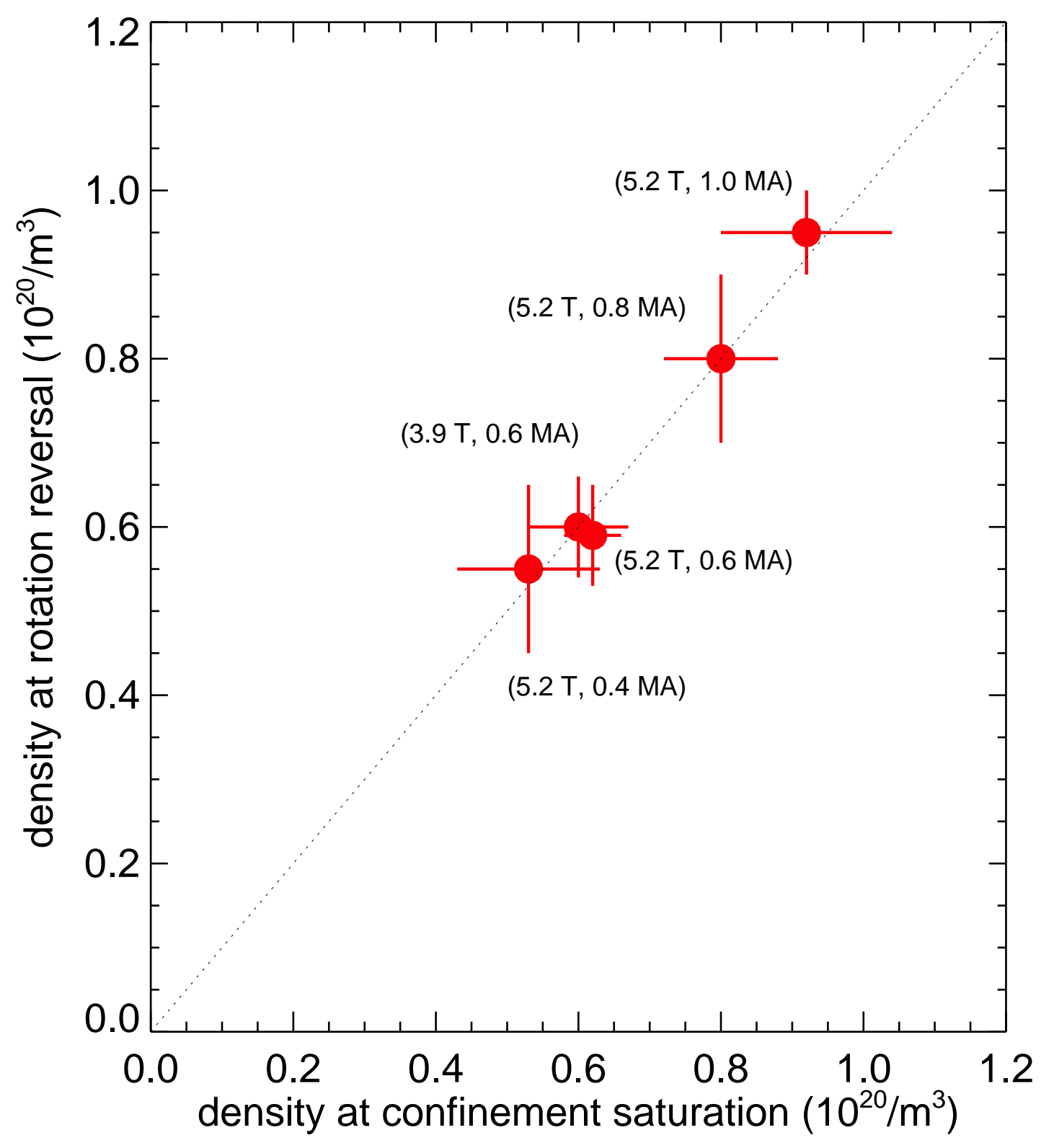

Figure $06 \quad$ LE13125 22Nov2011 